\title{
Elastic and buckling analysis of trapezoidal support diaphragms in steel box girder bridges
}

\author{
W. S. S. SIMONIAN \& F. SAWKO
}

\section{Mr B. G. R. Holloway, Consulting Engineer}

There is much more analytical work to be done before the designer, who cannot afford the time and expense of the extensive trial and error fundamental analysis described in the Paper, can apply the results to box girder bridge design.

41. I would like to suggest that the following aspects are investigated further.

(a) The effect of unsymmetrical live loading about both axes in plan.

(b) The varying requirements for web thickness and slope of the horizontal stiffeners, with increasing side slopes. The webs would appear to be related to the shear boot effect on the diaphragm webs.

(c) The effect on local stresses due to the elastic deformations of the diaphragm under load where bearings of significant length are provided. Provision can be made to mitigate this effect under the dead loading but it does not appear practical to do this under live loading unless spherical bearings are provided in conjunction with rollers or flexible piers.

(d) The effect of varying spacing of the bearings in relation to the width of the lower box girder flange.

42. It might then be possible to relate the Authors' findings to the Merrison rules and to propose a number of empirical rules to help the hard-pressed designer determine the initial sections. It is appreciated, however, that it may still be necessary ultimately to complete a finite element analysis as a check.

43. What do the Authors mean by the term 'continuum mechanics' in $\$ 1$ ?

\section{Dr Simonian and Professor Sawko}

The main aim of the present work was to establish a general method of analysis capable of dealing with all aspects of the problem including those in $\$ 41(a)-(d)$. The evolution of empirical rules is a far greater task, not because of any inherent difficulty, but because of the number of variables involved.

45. The term 'continuum mechanics' in the context of $\S 1$ relates to problems in which continuity of stress is maintained and the boundary conditions permit the evaluation of a closed solution (e.g. in the form of an infinite series or a polynomial). Such solutions exist for a number of cases of uniform plates with simple boundary conditions. The presence of stiffeners leads to stress discontinuities and in these situations numerical methods have to be used to provide an approximate solution. This latter case applies to stiffened box girder bridges. 